\title{
Propuesta agro-didáctica en la enseñanza de la bioestadística mediada por $\mathrm{TICs}^{1}$
}

\author{
María Claudia Carabalí ${ }^{2}$ \\ Diana Johana Carabalí-Banguero ${ }^{3}$ \\ Ervin Andrés Carabalí Caracas ${ }^{4}$
}

Recibido: 12-02-2018

Aceptado: 23-04-2018

\section{RESUMEN}

Se presenta una propuesta pedagógica cuya finalidad es enseñar conceptos estadísticos y biológicos con una práctica de aprendizaje constructivista bajo un modelo pedagógico cooperativo colaborativo. En la etnogranja de la I.E. Agroindustrial Valentín Carabalí, Buenos Aires (Cauca). Se estableció un cultivo de rábano sembrado en tres distancias de siembra, mediante la construcción de camas hortícolas. Los estudiantes realizaron el manejo agronómico con la asesoría de un grupo de docentes, a los 30 días se evaluó el efecto de la densidad de siembra mediante el software MINITAB versión 16 y Excel 2013. Los estudiantes desarrollaron competencias en la interpretación y representación. Esta estrategia didáctica permitió aplicar los pasos del método científico en el

1. Artículo resultado de investigación. Este producto se adscribe dentro de la propuesta pedagógica SABERES MATEMÁTICOS Y ENTOMOLÓGICOS DEL ENTORNO "MEDIADOS POR TIC" EN LA I.E. AGROINDUSTRIAL VALENTÍN CARABALI SEDE PRINCIPAL que adelantan docentes de la Institución Educativa Agroindustrial Valentín Carabalí Sede Principal ubicada en el corregimiento La Balsa, Municipio Buenos Aires (Cauca, Colombia) y es apoyado por la institución educativa.

2. Especialista en Didáctica de las Matemáticas (Universidad Libre). Licenciada en Matemáticas y Computación (Universidad Antonio Nariño). Docente de la Institución Educativa Agroindustrial Valentín Carabalí Sede Principal, Cauca, Colombia.

Correo: carabalimariaclaudia84@gmail.com

ORCID: https://orcid.org/0000-0003-3015-5583

3. Magister en Ciencias-Biología (Universidad del Valle). Bióloga (Universidad del Valle). Docente de la Institución Educativa Agroindustrial Valentín Carabalí Sede Principal, Cauca, Colombia.

Correo: dianajohana16@hotmail.com

ORCID: https://orcid.org/0000-0001-5383-4357

4. Especialista en Administración de la Informática Educativa (Universidad de Santander). Licenciado en Educación Rural (Centro Universitario de Bienestar Rural FUNDAEC). Docente de la Institución Educativa Agroindustrial Valentín Carabalí Sede Principal, Cauca, Colombia. Correo: ervina68@hotmail.com

ORCID: https://orcid.org/0000-0001-9744-3710 
aprendizaje vivencial del concepto de competencia intraespecífica, en el cual el uso de herramientas informáticas es fundamental.

Palabras clave: Competencia intraespecífica, Biología, Estadística, Hortalizas, MINITAB.

\title{
Agro-didactic proposal in the teaching of biostatistics mediated by ICTs
}

\begin{abstract}
A pedagogical proposal is presented whose purpose is to teach statistical and biological concepts with a constructivist learning practice with a collaborative cooperative pedagogical model. In the ethnofarm of the I.E. Agroindustrial Valentín Carabalí, Buenos Aires (Cauca). A cultivation of radish was planted in three planting distances it was established by the construction of horticultural beds. The students performed the agronomic management with the advice of a group of teachers. 30 days later, the seed density effect was evaluated using software MINITAB version 16 and Excel 2013. Students developed competencies in interpretation and representation. This didactic strategy allowed to apply the steps of the scientific method in the experiential learning of the concept of intraspecific competition, in which the use of computer tools is fundamental.
\end{abstract}

Keywords: Interspecific Competition, Biology, Statistics, Vegetables, MINITAB.

\section{Proposition agro-didactique dans l'enseignement de la biostatistique par les TIC}

\section{RÉSUMÉ}

On présente une proposition pédagogique qui s'agit d'enseigner concepts statistiques et biologiques avec une pratique d'apprentissage constructiviste selon le model pédagogique collaboratif et coopératif. 
Dans l'ethnographie de I.E. agroindustriel Valentín Carabalí, Buenos Aires (Cauca). Une culture de radis s'est établie en trois distances de semaille, grâce à la construction de lits horticoles. Les étudiants ont fait la part agronomique avec l'aide d'un groupe de professeurs, 30 jours après ils ont évalué l'effet de la densité de la semaille à l'aide d'un software MINITAB version 16 et Excel 2013. Les étudiants ont développé compétences d'interprétation et représentation. Cette stratégie didactique a permis appliquer les pas de la méthode scientifique dans l'apprentissage existentiel du concept de concurrence intraspécifique, dans lequel l'usage de outils informatiques est fondamental.

Des mots clés: concurrence intraspécifique, biologie, légumes, MINITAB.

\section{Introducción}

El rábano Raphanus sativus L. var. Crimson Giant. (Brassicaceae) es una hortaliza anual, originaria de China o Japón, que de acuerdo a la variedad presenta raíces gruesas de coloración roja, rosado o blanco que varían en tamaño y forma (Africano y Pinzón 2014). Es rica en vitaminas A, B1, B2 y $C$, al igual que en minerales. También tiene propiedades nutracéuticas (Criollo y García, 2009; Africano y Pinzón 2014). Su ciclo de desarrollo es corto, entre 20 y 70 días de acuerdo a la variedad (Criollo y García, 2009). Se adapta a cualquier tipo de suelos, siendo su crecimiento ideal en los suelos con pH ligeramente ácido entre 5,5 y 6,8 y en temperaturas entre los $15-18^{\circ} \mathrm{C}$ (Africano y Pinzón 2015).

En los agroecosistemas los recursos son limitados; las plantas compiten por la energía solar, el espacio, el agua, los nutrientes. Esta competencia se evidencia en el desarrollo de las plantas ya que se reduce la aptitud o el éxito reproductivo de las plantas en competencia, siendo las plantas que crecen menos rápido o tienen menor acceso a los recursos las más afectadas (Curtis y Schnek 2008).

La productividad del cultivo de rábano y crecimiento de las plantas depende de las características genotípicas y el ambiente donde se desarrollen influencia el fenotipo de las plantas (Criollo y García, 2009). La distancia de siembra es una práctica agrícola que pretende obtener un rendimiento óptimo del cultivo en términos de: número de hojas, 
diámetro del bulbo, biomasa y área foliar. Cuando la distancia de siembra es óptima, las plantas utilizan de manera eficiente los recursos. Por el contrario, densidades de siembra inadecuadas conllevan al incremento de la incidencia de plagas, e incrementan la competencia por la energía, y los nutrientes disponibles en el suelo (Criollo y García, 2009).

La educación en ciencias naturales y matemáticas tradicionalmente ha sido abordada desde un modelo educativo academicista, en el cual se prioriza más en la enseñanza y la memorización de conceptos que en la apropiación y comprensión de los mismos (Martínez y Torres 2013). Frente a este modelo, el constructivismo se enfoca en el aprendizaje significativo, en el cual el docente es un facilitador o moderador de la interacción con el medio permite la construcción del conocimiento (Martínez y Torres 2013). Los estudiantes participan de manera dinámica bajo un enfoque de aprendizaje cooperativo-colaborativo interactuando con sus compañeros y construyendo sus propios conocimientos y saberes (Onrubia et al. 2008). El análisis de plasticidad fenotípica de esta planta bajo distintas densidades de siembra permite enseñar conceptos biológicos, estadísticos y trigonométricos, al calcular el peso seco de las plantas y su área foliar. El análisis del efecto de la densidad de siembra en la productividad de las plantas permite lograr una mayor comprensión del estudiantado de este concepto y el de competencia intraespecífica, al determinar mediante práctica de campo el efecto de una sola variable en la productividad del cultivo.

El objetivo de esta investigación fue emplear el cultivo de rábano como modelo para la enseñanza-aprendizaje de estadística descriptiva, conceptos biológicos y trigonometría empleando las TIC en estudiantes del grado $10^{\circ}$ de la Institución Educativa Agroindustrial Valentín Carabalí (Aparicio \& Ostos, 2018).

\section{Metodología}

\section{Evaluación del rendimiento}

Se llevó a cabo la siembra de semillas de rábano Raphanus sativus variedad Crimson Giant bajo condiciones de campo en la etnogranja la Valentina (Coordenadas). Se ubicaron en las eras, tres niveles de densidad de siembra: densidad baja, media y alta con $10 \mathrm{~cm}, 5 \mathrm{~cm}$ y $3 \mathrm{~cm}$ de separación entre sí respectivamente, en un área de $1 \mathrm{~m} 2$ para 
cada tratamiento. Se dejó un espacio de $0,5 \mathrm{~m} 2$ para disminuir el efecto borde. Utilizando plantillas plásticas que tenían un patrón de huecos correspondiente a cada nivel de densidad de siembra y un medidor de profundidad se sembraron las semillas en un patrón hexagonal a $1 \mathrm{~cm}$ de profundidad cada una. Las plantas fueron regadas día de por medio durante el tiempo que duró el experimento.

A la cuarta semana de haber realizado la siembra, se midió el largo y ancho de la hoja más grande. Posteriormente, en la quinta semana se cosecharon las parcelas tomando las muestras con base en el número de plantas encontradas en el nivel de baja densidad. Se evaluó el efecto de la densidad de siembra teniendo en cuenta peso húmedo de la raíz, peso húmedo de las hojas, número de hojas por planta, diámetro de la raíz y área foliar.

Se realizó un diseño en bloques al azar con 4 repeticiones. Los datos fueron analizados mediante un análisis de varianza (ANOVA), posteriormente se utilizaron pruebas de comparación de medias de Tukey $(P \leq 0,05)$ con el software MINITAB versión 16 (Minitab, State College, PA, EEUU), para determinar entre cuales variables de respuesta había mayor diferencia.

\section{Estrategia pedagógica}

La población estuvo representada por tres docentes y 19 estudiantes del grado $10^{\circ}$ de media técnica y 21 estudiantes del grado $6^{\circ}$ de básica secundaria de la Institución Educativa Agroindustrial Valentín Carabalí, Sede Principal, del corregimiento de La Balsa, municipio de Buenos Aires (Cauca). Las prácticas agrícolas de: siembra, riego, fertilización, manejo fitosanitario de las plantas fueron orientadas por los docentes, para permitir que los estudiantes desarrollaran un trabajo cooperativocolaborativo bajo un modelo constructivista (Aparicio \& Ostos, 2018). Los estudiantes registraron los datos de cosecha en un formato y realizaron la transposición didáctica, y el análisis de la información con estadística descriptiva mediante tablas de distribución de frecuencias, porcentajes y análisis de varianza, los estudiantes recibieron la asesoría de los docentes.

Los alumnos de acuerdo a sus preconceptos e información suministrada en el aula de clase, relacionan con lo observado en la práctica, con la intención de lograr un aprendizaje significativo (Langer, 2016). El empleo de estrategias didácticas como la lectura de textos 
académicos relacionados con el tema, lectura e interpretación de símbolos matemáticos, estadísticos y trigonométricos, el uso de la tecnología para la toma de decisiones con base en el análisis de la información, se recopiló esta información, se sistematizó y se analizó de acuerdo a los objetivos de la investigación (de la Calle et al., 2014).

\section{Resultados}

\section{Número de hojas}

Se presentaron diferencias estadísticamente significativas en el número de hojas producidas por planta bajo tres densidades de siembra distintas (ANOVA $F=13,48 ;$ g.l. $=2 ; P=0,000$ ). Las plantas sembradas a baja densidad de siembra presentaron mayor número de hojas $(\square=7,6 \pm S . E .=0,2)$, no se presentaron diferencias en el número de hojas en la densidad de siembra media $(\square=6,7 \pm 0,2)$ y alta $(\square=6,1 \pm 0,2)$. No se presentaron diferencias entre los bloques (ANOVA $F=0,44 ; g . I .=2 ; P=0,644$ )

Figura 1. Número promedio de hojas en plantas de rábano Raphanus sativus bajo tres densidades de siembra.

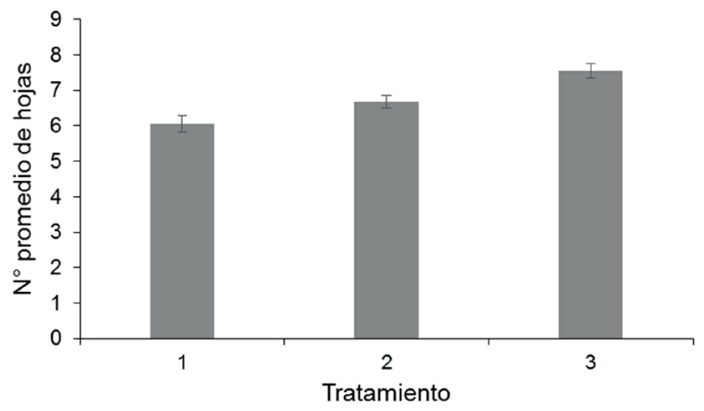

Fuente. Elaboración propia.

\section{Altura de la planta}

La mayor altura de las plantas se observó en el tratamiento de alta densidad de siembra (ANOVA $F=18,91 ;$ g.l. $=2 ; P=0,000$ ). No se encontraron diferencias en los tratamientos de densidad de siembra media $(\square=29,8 \pm 1,35)$ y baja $(\square=32,9 \pm 0,68)$, estos mostraron promedios similares. No se presentaron diferencias estadísticamente significativas entre los bloques (ANOVA F=0,26; g.I. =2; $P=0,771$ ). 
Figura 2. Altura promedio de plantas de rábano Raphanus sativus bajo tres densidades de siembra.

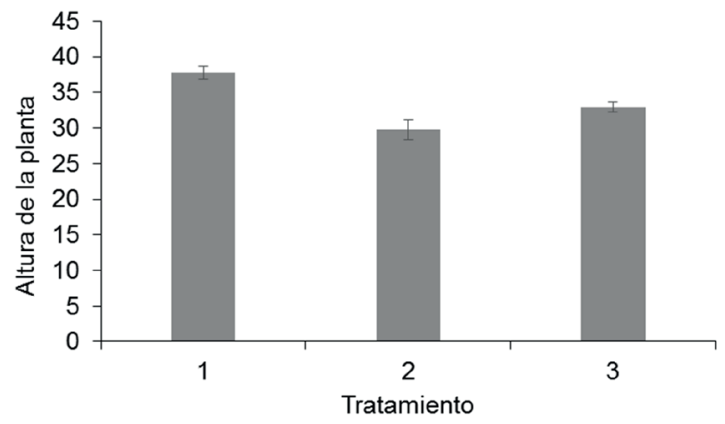

Fuente. Elaboración propia.

\section{Ancho de las hoja más grande}

Se presentaron diferencias entre los bloques (ANOVA $F=10,23 ;$ g.l.=2; $P=0,000)$. No se encontraron diferencias en el ancho de la hoja principal (ANOVA $\mathrm{F}=0,90 ;$ g.l. $=2 ; \mathrm{P}=0,407$ ). Esta variable mostró promedios similares en los tratamientos de alta $(\square=8,7 \pm 0,2)$, media $(\square=9,1 \pm 0,3)$ y baja $(\square=9,2 \pm 0,2)$ densidad de siembra.

Figura 3. Ancho promedio de la hoja más grande de plantas de rábano Raphanus sativus bajo tres densidades de siembra.

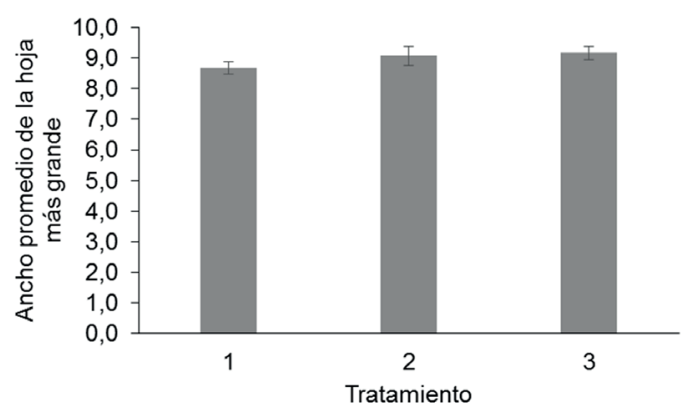

Fuente. Elaboración propia.

\section{Área Foliar}

Se presentaron diferencias entre los bloques (ANOVA $F=4,07 ;$ g.l.=2; $P=0,019)$. Se presentaron diferencias estadísticamente significativas en el área foliar (ANOVA $F=4,27 ;$ g.I. $=2 ; P=0,015$ ). En el tratamiento de alta densidad de siembra se obtuvo la mayor área foliar (Tukey, $P \leq 0,05$ ). En el T-1 las plantas tuvieron un área promedio de $(\square=330,12 \pm 12,4)$, en el T-2 de $(\square=287,2 \pm 18,4)$ y $\mathrm{T}-3$ de $(\square=303,3 \pm 10,9)$. 
Figura 4. Área foliar de plantas de rábano Raphanus sativus bajo tres densidades de siembra.

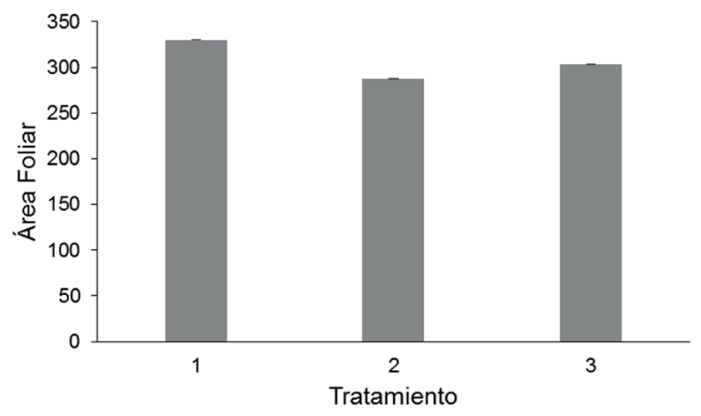

Fuente. Elaboración propia.

\section{Peso de la raíz}

Se presentaron diferencias entre los bloques (ANOVA $F=4,82 ;$ g.l. $=2$; $\mathrm{P}=0,009$ ), Las plantas mostraron diferencias en el peso de la raíz (ANOVA $F=14,87 ; g . I .=2 ; P=0,009$ ). Las raíces más livianas se dieron en el tratamiento de alta densidad de siembra $(\square=12,5 \pm 1,5)$, no se presentaron diferencias significativas estadísticamente entre los tratamientos de densidad media $(\square=26,8 \pm 2,7)$ y baja $(\square=26,9 \pm 2,2)$ (Tukey, $P \leq 0,05)$.

Figura 5. Peso promedio de la raíz de plantas de rábano Raphanus sativus bajo tres densidades de siembra.

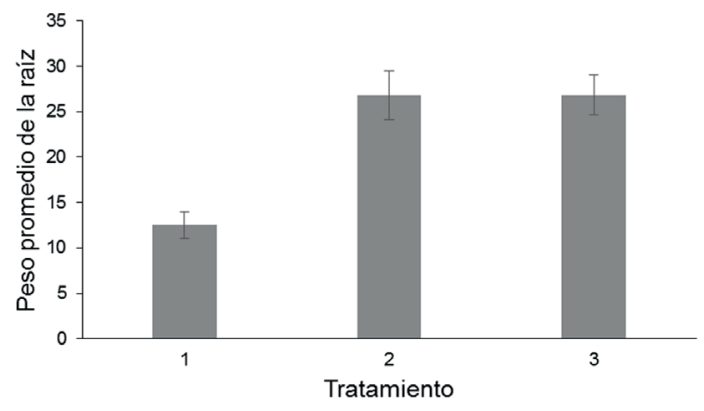

Fuente. Elaboración propia.

\section{Peso húmedo de las hojas}

Se presentaron diferencias entre los bloques (ANOVA $\mathrm{F}=4,65$; g.l. $=2$; $P=0,011$ ). No se presentaron diferencias en el peso de las hojas (ANOVA $\mathrm{F}=2,84 ; \mathrm{g} . \mathrm{I}=2 ; \mathrm{P}=0,061)$. Esta variable mostró promedios similares en los tratamientos de alta $(\square=28,9 \pm 1,6)$, media $(\square=34,2 \pm 2,3)$ y baja $(\square=35,6 \pm 2,1)$. 
Figura 6. Peso húmedo promedio de las hojas de plantas de rábano Raphanus sativus bajo tres densidades de siembra.

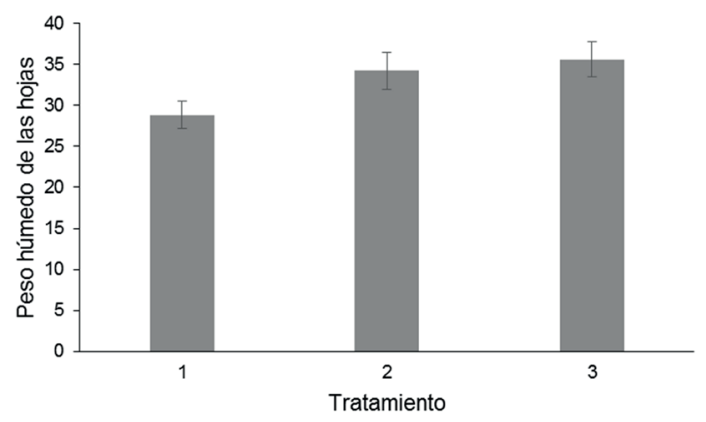

Fuente. Elaboración propia.

\section{Diámetro de la Raíz}

Se presentaron diferencias significativas en el diámetro de la raíz entre los tratamientos (ANOVA F $=5,42 ;$ g.l. $=2 ; \mathrm{P}=0,005$ ). En el tratamiento de alta densidad de siembra se obtuvo el menor diámetro de las raíces $(\square=8,1 \pm 0,4)$, comparado con densidad de siembra media $(\square=12,3 \pm 1,5)$ y baja $(\square=12 \pm 0,4)$.

Figura 7. Diámetro promedio de las raíces de plantas de rábano Raphanus sativus bajo tres densidades de siembra.

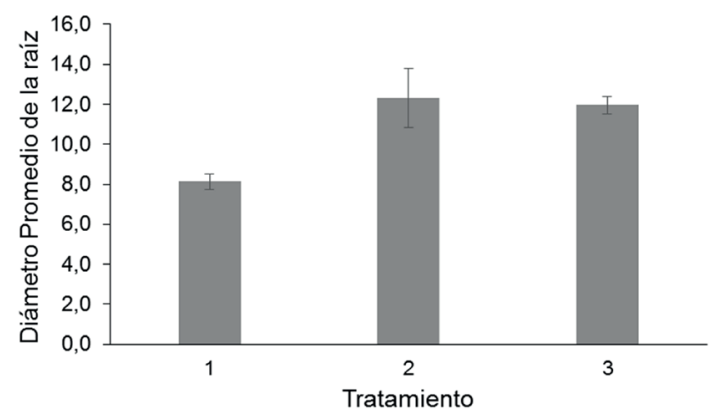

Fuente. Elaboración propia.

Las prácticas agrícolas impartidas en la institución se fundamentan en algunos métodos convencionales integrados con prácticas etnoculturales o ancestrales de la zona (Pérez, 2013). Se utilizan algunos agroquímicos para el control de plantas arvenses y plagas, no obstante se realiza la incorporación de abonos orgánicos, labranza mínima con la finalidad de generar conciencia ambiental con que favorezca la conservación de la biota, el suelo y el agua. También se constituyó en una herramienta para fortalecer el proceso de soberanía alimentaria, al fomentar el consumo de productos de alta calidad nutricional, saludables y prácticas agrícolas 
de bajo impacto ambiental (Viejo, Cabezas \& Martínez, 2013). Por otra parte, Los estudiantes confrontaron la información suministrada en el aula de clases con las observaciones y conclusiones que obtuvieron en el campo. Se demostró la aplicabilidad de los conceptos matemáticos en las actividades agrícolas, como lo son la estadística descriptiva y la demostración de teoremas trigonométricos.

Los estudiantes mostraron disposición por adquirir conocimientos de las distintas áreas integradas en la ejecución de la propuesta pedagógica (Ferreyra, 2014), cultivando la tierra y enseñando a estudiantes del grado 6 sobre las actividades planteadas, estos mostraron interés y motivación al aprender de manera diferente a la que se imparte tradicionalmente en el aula de clases.

\section{Discusión}

La densidad de la población tiene un efecto en el desarrollo de las plantas. Las plantas sembradas en el tratamiento de alta densidad mostraron un crecimiento mayor respecto a los otros tratamientos, debido al limitado espacio, y un rápido crecimiento que le permite a la planta alcanzar mayor superficie fotosintética al asimilar la mayor cantidad de los recursos disponibles (Criollo y García, 2009). Se corroboró lo encontrado por Criollo y García (2009) en cultivos de rábano en invernadero, en el cual un mayor número de plantas/área generó menor crecimiento de las raíces. Las plantas en el tratamiento de alta densidad tuvieron una competencia intraespecífica mayor por los nutrientes, espacio y luz solar, lo que limitó el desarrollo radicular. En resumen, el área foliar fue mayor ya que la planta requiere interceptar la radiación solar. Sin embargo la bioacumulación de fotoasimilados en las raíces fue baja.

En el tratamiento de baja densidad de siembra hay menor competencia por los recursos disponibles, por lo cual se incrementa la tasa de asimilación de nutrientes y la bioacumulación en la raíz (Criollo y García, 2009). Las plantas sembradas en el tratamiento de baja densidad tuvieron un mayor número de hojas. Pérez de Camacaro et al. 2005 indican que variables como el número de hojas y el peso de la planta se incrementan en este tratamiento. Las diferencias en el crecimiento de las plantas de rábano debido a la densidad de siembra pudieron ser evaluadas por los alumnos, quienes tuvieron aprendizajes significativos. 
La propuesta pedagógica es pertinente y contextualizada con las necesidades de capacitación de los estudiantes y se integran las tecnologías de la información para que el estudiante esté en la capacidad de responder en un mundo cada vez más globalizado (Pérez, 2014). Se hace énfasis en que se debe promover la enseñanza de técnicas agrícolas amigables con el medio ambiente que conlleven a mantener la productividad del suelo, recurso hídrico y conservar la flora y fauna asociada (Mujica et al. 2015). En la construcción de las eras se implementa el modelo pedagógico constructivista al utilizar el terreno disponible en la etnogranja para la producción de hortalizas, en este espacio se fortalecen los conocimientos adquiridos dentro del aula ya que el estudiante, guiado por el docente, es gestor de su proceso de aprendizaje (Konieczny, 2015). De esta manera se fomenta el desarrollo de competencias al adquirir experiencias que los motivan e incrementan su potencial con el trabajo cooperativo-colaborativo (Onrubia et al. 2008).

El trabajo cooperativo-colaborativo es una estrategia en la cual mediante la interacción con los compañeros de clase y docentes, se busca que los estudiantes sean personas más responsables, participativas y mejoren las competencias comunicativas, tanto en el proceso de lectoescritura realizando un trabajo productivo como en la convivencia (Onrubia et al. 2008).

Las actividades de campo permitieron el desarrollo de los pasos del método científico. Los estudiantes se preguntaron sobre si afecta la distancia entre plantas el desarrollo de las mismas, en respuesta a esta inquietud se plantearon hipótesis sobre cuál sería la distancia de siembra más favorable para el desarrollo de las plantas y obtener una mayor productividad, considerando que la mayor distancia de siembra favorecería el desarrollo de las plantas de acuerdo a los conceptos aprendidos en el aula de clases.

La experimentación permitió realizar observaciones e inferir sobre lo observado en campo. En el análisis de la información, con la asesoría de los docentes los estudiantes mejoraron sus habilidades en el área de la informática al transcribir la información, construir gráficos y usar software estadístico y trigonométrico, mediante estas estrategias didácticas los docentes logramos que los estudiantes adquieran competencias básicas, laborales y la socialización de los resultados obtenidos (Sabino 2014). Los pasos del método científico fundamentan las competencias específicas en ciencias naturales, como lo son: la capacidad de identificar fenómenos, 
indagar o plantear preguntas sobre lo observado, la comprensión, la capacidad de compartir conocimientos aceptando puntos de vista distintos a los propios e interactuando productivamente con el trabajo en equipo (MEN 2003).

Los estudiantes elaboraron sus conocimientos a partir de las actividades guiadas por los docentes y el empleo de las TIC, estas herramientas son fundamentales al permitirles adquirir gran cantidad de información, determinar el software más adecuado para su objetivo y corroborar o replantear sus conocimientos previos (Martínez y Torres 2013).

\section{Conclusiones}

Los métodos pedagógicos implementados facilitaron la adquisición del conocimiento y el uso de las TIC. Se fortalecieron las habilidades tecnológicas al utilizar las herramientas computacionales disponibles en la institución para resolver un problema del contexto. De igual manera se fomentó el trabajo en grupo, se generaron aprendizajes significativos y se desarrollaron competencias laborales con las cuales los estudiantes pueden capacitar a otros miembros de su comunidad de manera que se participan en aspectos socioeconómicos comunitarios. Dentro de la dinámica del proceso de paz la seguridad alimentaria contribuye a generar convivencia entre los estudiantes. A su vez la producción de alimentos posibilita un mayor acercamiento de estos, genera nexos para crear estrategias de mercadeo o intercambio con impacto en la comunidad. Finalmente la información puede ser analizada, sintetizada y socializada empleando las TIC.

\section{Referencias bibliográficas}

Aparicio, O.Y., \& Ostos, O.L. (2018). Las TIC como herramientas cognitivas para la investigación. Revista Interamericana de Investigación, Educación y Pedagogía, RIIEP, 11(1). https://orcid.org/0000-0003-3535-6288. https://orcid.org/0000-0002-64779872

Africano Peréz, K. L., \& Pinzón Sandoval, E. H. (2015). Comportamiento fisiológico de plantas de rábano (Raphanus sativus L.) sometidas a estrés por salinidad. Conexión Agropecuaria JDC, 4(2), 11-22. 
Criollo, H. y García, J. (2009). Efecto de la densidad de siembra sobre el crecimiento de plantas de rábano (Raphanus sativus L.) bajo invernadero. Revista Colombiana de Ciencias Hortícolas 3(2): 210-222. Recuperado de http://revistas.uptc.edu.co/index. php/ciencias_horticolas/article/view/1214

Curtis, H. y Schnek, A. (2008). Curtis Biología. Editorial Médica Panamericana. Séptima Edición en Español. 1160 p.

de la Calle, C. V., Malaver, M. O., Gallego, J. D. M., Rodríguez, M., Flórez, J. C., Henao, C. E. \& Saldaña, R. (2014). Aportes de los doctorados de educación en ciencia, tecnología y sociedad, desde la sistematización de sus investigaciones doctorales científicas y formativas, 2000-2010. Revista Interamericana de Investigación, Educación y Pedagogía, RIIEP, 7(1). DOI: https://doi.org/10.15332/s1657-107X.2014.0001.04

Ferreyra, H. A. (2014). Mesas Socioeducativas para la Inclusión y la Igualdad. Un programa "De todos con todos". Una experiencia en construcción. Revista Interamericana de Investigación, Educación y Pedagogía, RIIEP, 7(2). DOI: https://doi. org/10.15332/s1657-107X.2014.0002.01

Konieczny, P. (2015). Lorenzo García Aretio: bases, mediaciones y futuro de la educación a distancia en la sociedad digital. Revista Interamericana de Investigación, Educación y Pedagogía, RIIEP, 8(1). DOI: https://doi.org/10.15332/s1657107X.2015.0001.08

Langer, E. (2016). La construcción de confianza para el estudio de prácticas de resistencia en la escolarización de jóvenes en contextos de pobreza urbana. Revista Interamericana de Investigación, Educación y Pedagogía, RIIEP, 9(2). DOI: https://doi. org/10.22490/25391887.1945

Martínez, A. y Torres, L. (2013). Los entornos personales de aprendizaje (PLE). Del cómo enseñar al cómo aprender. Revista Educación Mediática y TIC 2(1): 39-57. Recuperado de http://www.uco.es/servicios/ucopress/ojs/index.php/edmetic/article/ view/2860/2752

Ministerio de Educación Nacional. MEN (2003). Estándares Básicos de Competencias en Ciencias Sociales y Ciencias Naturales. Santafé de Bogotá. Recuperado de https://www. mineducacion.gov.co/1621/articles-340021_recurso_1.pdf

Mujica, H., Suárez, M. y Rodríguez, A. (2015). Aula agroecológica. Alternativa de enseñanza para escuelas rurales. SABER. Revista Multidisciplinaria del Consejo de Investigación de la Universidad de Oriente ene-mar 27(1): 120-129. Recuperado de http://www.redalyc.org/pdf/4277/427739474015.pdf

Onrubia, J., Colomina, R. y Engel, A. (2008). Entornos Virtuales de aprendizaje basados en el trabajo en grupo y el aprendizaje colaborativo. En Coll, C. y Monereo, C. (Eds.). Psicología de la educación virtual: aprender y enseñar con las tecnologías de la información y la comunicación (pp. 213-231). Madrid: Ediciones Morata. 
Pérez, T. H. P. (2013). Aproximaciones al estado de la cuestión de la investigación en educación y derechos humanos. Revista Interamericana de Investigación, Educación y Pedagogía, RIIEP, 6(1). DOI: https://doi.org/10.15332/s1657-107X.2013.0001.05

Pérez, T. H. (2014). Colombia: de la educación en emergencia hacia una educación para el posconflicto y la paz. Revista Interamericana de Investigación, Educación y Pedagogía, RIIEP, 7(2). DOI: https://doi.org/10.15332/s1657-107X.2014.0002.06

Pérez de Camacaro, M., Carew L. y Battey N. (2005). Efecto de la densidad de siembra sobre el crecimiento vegetativo y reproductivo de la fresa cv Elsanta. Bioagro 17(1): 11-15.

Sabino, C. (2014). El proceso de investigación. Guatemala: Editorial Episteme.

Viejo, C. M., Cabezas, I. L., \& Martínez, M. D. J. I. (2013). Las redes de académicas en la docencia universitaria. Revista Interamericana de Investigación, Educación y Pedagogía, RIIEP, 6(2). DOI: https://doi.org/10.15332/s1657-107X.2013.0002.03 